\title{
CORANKS OF A QUASI-PROJECTIVE MODULE AND ITS ENDOMORPHISM RING
}

\author{
by TSUTOMU TAKEUCHI
}

(Received 9 March, 1993)

Recently several authors have studied dualizing Goldie dimension of a module: spanning dimension in [2], codimension in [13], corank in [16] and also $[9,17,12,5,11,6$, 4, 7] ([13] may be read in comparison with the others). In the present note we prove the equality corank ${ }_{R} P=\operatorname{corank}_{S} S$, where $P$ is a quasi-projective left $R$-module and $S$ is its endomorphism ring. This result is an answer to the question [12, p. 1898] and an extension of $[3$, Corollary 4.3] which shows the above equality for a $\Sigma$-quasi-projective left $R$-module $P$.

Throughout what follows $R$ denotes an associative ring with identity and ${ }_{R} P,{ }_{R} M$ left $R$-modules. Let $S$ be the endomorphism ring of ${ }_{R} P$. Then $P$ is an $(R, S)$-bimodule: $P={ }_{R} P_{S}, P^{*}:=\operatorname{Hom}_{R}(P, M)$ is a left $S$-module and

$$
P^{*}(A):=\left\{f \in P^{*} \mid P f \subset A\right\}
$$

is a submodule of ${ }_{S} P^{*}$ for a submodule ${ }_{R} A$ of ${ }_{R} M$. For the definitions and properties we refer to [13] on coindependency of a set of submodules (which accords with mèetindependency in [3]), to [16] on corank of a module and to [10] on a $\Sigma$-quasi-projective module. A set $\mathscr{S}$ of proper submodules of $M$ is said to be coindependent if $\bigcap_{i=1}^{n-1} A_{i}+A_{n}=$ $M$ for any finite subset $\left\{A_{1}, A_{2}, \ldots, A_{n}\right\}$ of $\mathscr{S}$ with $n \geqq 2$. Every single-element set is coindependent. We say corank ${ }_{R} M=n$, a positive integer, if there exists an epimorphism from $M$ to a direct product of $n$ nonzero modules but there is no epimorphism from $M$ to one of $n+1$ nonzero modules. Then corank ${ }_{R} M=n$ if and only if there exists a coindependent set of $n$ proper submodules of $M$ but there is no coindependent set of $n+1$ proper ones. A module $M$ is said to be $\Sigma$-quasi-projective if the direct sum of any number of copies of $M$ is quasi-projective. It is said that $P$ (finitely) generates $M$, or $M$ is (finitely) $P$-accessible, if $M$ is a homomorphic image of a (finite) direct sum of copies of $P$. It is also said that $P$ is finitely $M$-projective if for every homomorphism $f$ of $P$ into $N$ and every epimorphism $h$ of $M$ onto $N$, with $N$ an arbitrary finitely cogenerated left $R$-module, there exists a homomorphism $g$ in $P^{*}$ such that $g h=f$ (see [15]).

As the first half of our preparation we borrow the following from [3, Theorem 4.2i)].

Proposition 1. If $P$ is quasi-projective and $P$ finitely generates $M$, then corank ${ }_{s} P^{*} \leqq$ $\operatorname{corank}_{R} M$.

Proof. See [3, p. 104] or [1, Section 4].

The second half of our preparation is a continuation of [14] and [15].

Lemma 2. Let $P$ be finitely $M$-projective and let $\left\{B_{1}, B_{2}, \ldots, B_{n}\right\}$ be a coindependent set of proper submodules of $M$ such that each $M / B_{i}$ is finitely cogenerated. Then for any

Glasgow Math. J. 36 (1994) 381-383. 
$f_{1}, f_{2}, \ldots, f_{n}$ in $P^{*}$ there exists a homomorphism $g$ in $P^{*}$ such that $g-f_{i}$ belongs to $P^{*}\left(B_{i}\right)$ for each $i$.

Proof. Set $\bar{M}:=\bigoplus_{i=1}^{n} M / B_{i}$ and define homomorphisms $f, h$ as follows:

$$
\begin{array}{lll}
f: P \rightarrow \bar{M}, & p f=\left(p f_{i}+B_{i}\right) & (p \in P), \\
h: M \rightarrow \bar{M}, & x h=\left(x+B_{i}\right) & (x \in M) .
\end{array}
$$

Then coindependency $B_{i}+\bigcap_{j \neq i} B_{j}=M$ implies that $h$ is an epimorphism. Since $\bar{M}$ is finitely cogenerated and $P$ is finitely $M$-projective, there exists a homomorphism $g$ in $P^{*}$ such that $g h=f$. Thus we have $P\left(g-f_{i}\right) \subset B_{i}$ for each $i$.

Proposition 3. If $P$ is finitely $M$-projective and $P$ generates $M$, then corank ${ }_{R} M \leqq$ corank ${ }_{s} P^{*}$.

Proof. Assume that $\left\{A_{1}, A_{2}, \ldots, A_{n}\right\}$ is a coindependent set of proper submodules of $M$. Then it is easily deduced from [8, Lemma 1.1], or proved directly that there exist proper submodules $B_{i}, A_{i} \subset B_{i}$, of $M$ such that each $M / B_{i}$ is finitely cogenerated. Evidently $\left\{B_{1}, B_{2}, \ldots, B_{n}\right\}$ is a coindependent set. Since $B_{i}$ is proper in $M=P P^{*}$, we know that each ${ }_{s} P^{*}\left(B_{i}\right)$ is a proper submodule of ${ }_{s} P^{*}$. Let $f$ be in $P^{*}$. Then Lemma 2 implies that for each $i$ there exists a homomorphism $g$ in $P^{*}$ such that

$$
g-f \in P^{*}\left(B_{i}\right) \text { and } g-0 \in P^{*}\left(B_{j}\right) \quad(j \neq i) .
$$

Thus $P^{*}\left(B_{i}\right)+\bigcap_{j \neq i} P^{*}\left(B_{j}\right)=P^{*}$ for each $i$ and hence $\left\{P^{*}\left(B_{1}\right), P^{*}\left(B_{2}\right), \ldots, P^{*}\left(B_{n}\right)\right\}$ is a coindependent set of proper submodules of $P^{*}$. This gives the conclusion.

Corollary 4 ([3, Theorem 4.2ii)]). If $P$ is $\Sigma$-quasi-projective and $P$ generates $M$, then corank ${ }_{R} M \leqq$ corank ${ }_{S} P^{*}$.

Proof. This is clear since every $\Sigma$-quasi-projective module that generates $M$ is $M$-projective.

Combining Propositions 1 and 3, we have the following.

THeOREM 5. If $P$ is quasi-projective and $P$ finitely generates $M$, then corank $_{R} M=$ corank ${ }_{s} P^{*}$.

COROLlaRy 6. If $P$ is quasi-projective, then corank ${ }_{R} P=\operatorname{corank}{ }_{S} S$.

ACKNOWLedgement. The author would like to thank the referee for his kind and constructive comments.

\section{REFERENCES}

1. T. Albu and C. Năstăsescu, Relative finiteness in module theory, (Marcel Dekker, 1984).

2. P. Fleury, A note on dualizing Goldie dimension, Canad. Math. Bull. 17 (1974), 511-517. 
3. J. L. García Hernández and J. L. Gómez Pardo, On endomorphism rings of quasiprojective modules, Math. Z. 196 (1987), 87-108.

4. P. Grzeszczuk and E. R. Puczyłowski, On Goldie and dual Goldie dimensions, J. Pure Appl. Algebra 31 (1984), 47-54.

5. A. K. Gupta and K. Varadarajan, Modules over endomorphism rings, Comm. Algebra 8 (1980), 1291-1333.

6. J. K. Haack, The duals of the Camillo-Zelmanowitz formulas for Goldie dimension, Canad. Math. Bull. 25 (1982), 325-334.

7. A. Hanna and A. Shamsuddin, Duality in the category of modules. Applications, Algebra Berichte 49, (Reinhard Fischer, 1984).

8. R. W. Miller and D. R. Turnidge, Factors of cofinitely generated injective modules, Comm. Algebra 4 (1976), 233-243. $255-262$

9. K. M. Rangaswamy, Modules with finite spanning dimension, Canad. Math. Bull. 20 (1977),

10. K. M. Rangaswamy, Some remarks on the endomorphism rings of quasi projective modules, Publ. Math. Debrecen 26 (1979), 71-73.

11. E. Reiter, A dual to the Goldie ascending chain condition on direct sums of submodules, Bull. Calcutta Math. Soc. 73 (1981), 55-63.

12. B. Sarath and K. Varadarajan, Dual Goldie dimension-II, Comm. Algebra 7 (1979), $1885-1899$.

13. T. Takeuchi, On cofinite-dimensional modules, Hokkaido Math. J. 5 (1976), 1-43. $29-31$

14. T. Takeuchi, A note on $M$-projective modules, Bull. Fac. Educ. Hirosaki Univ. 44 (1980), $168-170$

15. T. Takeuchi, A note on $M$-projective modules. II, Proc. Japan Acad. (A) 60 (1984),

16. K. Varadarajan, Dual Goldie dimension, Comm. Algebra 7 (1979), 565-610.

17. K. Varadarajan, Modules with supplements, Pacific J. Math. 82 (1979), 559-564.

Department of Mathematics

FACUlTY OF EDUCATION

Hirosaki University

HIROSAKI, 036

JAPAN 